\title{
Stiefkind der Diskussion
}

\begin{abstract}
Seit Jahren wird die Auseinandersetzung über sustainable development intensiv geführt. Der Bereich, der dabei die geringste Weiterentwicklung erfahren hat, ist soziale Nachhaltigkeit (1). Die Dringlichkeit zeigt sich am deutlichsten am Beispiel einer nachhaltigen Produktpolitik. Anstöße zu einer verstärkten Berücksichtigung sind am ehesten durch entsprechende Ratings sowie produktbezogene Dialogverfahren zwischen Unternehmen und Stakeholdern zu erwarten.
\end{abstract}

$\mathrm{N}$ Von Burghard Flieger und Elmar Sing achhaltigkeit bedeutet im folgenden die integrative Bearbeitung der Konflikte und Synergien zwischen sozialen, ökologischen und ökonomischen Dimensionen unternehmerischen Handelns. Nachhaltigkeit erscheint überwiegend als eine Nebenbedingung der Produkte und der Produktion eines Unternehmens: Trotz ihrer schädlichen Wirkungen soll die Regenerierungsfähigkeit der Erde gewährleistet bleiben. Demnach ist Nachhaltigkeit kein Optimierungsprozess, sondern ein Konzept zur Sicherung der Regeneration. Ähnlich wie im ökologischen Bereich wird auch im sozialen Bereich keine Optimierung im Sinne einer optimalen sozialen Bedürfnisbefriedigung angestrebt, sondern nur - soweit ökonomisch machbar - eine Bedürfnisberücksichtigung und Kompensation für soziale Schädigungen.

Veranschaulichen lässt sich dies an den häufig negativen Folgen von beruflicher Arbeit für soziale Primärgruppen wie die Familie. Dazu gehört die fehlende Zeit für die Erziehung und Betreuung von Kindern oder auch Stressphänomene infolge der Arbeit. Sie wirken unmittelbar in den privaten Bereich hinein durch Verhaltensweisen wie Streit (seinen Frust ablassen) oder Ersatzhandlungen (Kompensationskonsum). Unternehmen bemühen sich teilweise um eine Abschwächung solcher Probleme, indem sie familienfreundliche Arbeitszeitregelungen ermöglichen oder Seminare zum Stressabbau anbieten. Dies sind aber nur Ausgleichsmaßnahmen, ohne eine Lösung der zugrunde liegenden Schwierigkeiten im Sinne einer Balance zwischen Arbeit und Privatleben. Für viele Unternehmen ist dies erforderlich, um langfristig auf ihre Mitarbeiter als zentrale Ressource zurückgreifen zu können. Insofern sichern entsprechende Nachhaltigkeitskonzepte nur, dass die (kapital-) verwertungsorientierte Wirtschaft sich langfristig nicht entsprechend der Marxschen Voraussagen selbst ihrer Grundlagen beraubt.

\section{- Komplexe Bedürfniseinbindung}

Wie weit die Berïcksichtigung von Anforderungen sozialer Nachhaltigkeit von einer Optimierung entfernt ist, lässt sich auch an den Grenzen nachhaltiger Produktpolitik aufzeigen. Güter und Dienstleistungen erfüllen neben ihrer Kernfunktion immer auch abstraktere themenspezifische und soziale Funktionen. Das Überraschungsei steht als besonders exzellent konzipiertes Beispiel dafür, welche Vielzahl an Bedürfnissen Marketingstrategen mit einem Produkt abzudecken versuchen: Süßigkeit, Überraschung, Erlebnis, Selbstbau, Sammelleidenschaft, Sammelsieger, Wert der Vollständigkeit etc.

Wenn ein Unternehmen tatsächlich die gleichen Bedürfnisse ökologisch und sozial konsequent erfüllen will, müsste es anstreben, seine eigene Selbstauflösung durch die langfristig selbstorganisierte Bedürfnisbefriedigung seiner Konsumenten zu realisieren. Die meisten Unternehmen betreiben aber wie beim Überraschungsei eine gegenteilige Marketingstrategie. Durch die Verknüpfung ihres Produktes mit möglichst vielen weiteren Zusatznutzen wird eine hohe dauerhafte Bindung an ein Produkt angestrebt, um so aus Sicht des Unternehmens im Idealfall unbegrenzte Nachkaufambitionen zu fördern.

\section{Ökologische und soziale Effizienz}

Hinzu kommt: Viele Unternehmen können sich die Berücksichtigung sozialer Aspekte ab einem bestimmten Niveau ökonomisch nicht mehr leisten. Ein analoges Dilemma auf der Konsumentenseite resultiert daraus, dass viele öko-effiziente Produkte elitären Charakter haben. Obwohl oder auch weil sie ökologisch effizient sind, kann sie sich infolge des hohen Preises nur eine kleine Gruppe kaufstarker Verbraucher leisten. Solche Widersprüchlichkeiten erfordern eine Reihe von Zusatzïberlegungen bei der Entwicklung sozialer
Kriterien, die ein Produkt berücksichtigen müsste, soll es nicht nur ökologisch, sondern auch sozial als effizient eingestuft werden:

- Entkopplung des Konsums von der Anerkennung eines Gruppendrucks bzw. Verknüpfen von sozialer Anerkennung für ökologisch-soziales Kaufverhalten;

- Entdeckung der Langsamkeit im Sinne des Veraltens der Produkte unter ökologischem, aber auch modischem Blickwinkel;

- Partizipation der und Kommunikation mit den Kunden im Hinblick auf Entwicklung, Anwendung und Entsorgung von Produkten;

- Vorzug der personalen und vor allem der interaktiven Kommunikation;

- Anhebung des Anspruchsniveaus wirtschaftlich schlechter gestellter Personengruppen im Hinblick auf ökologisch unbedenkliche Produkte und Dienstleistungen;

- stufenweise Ausdehnung dieser Aspekte auf die sogenannte Dritte Welt.

\section{Anstöße durch Ratings}

Soziale Nachhaltigkeit und ihre Überprüfung beschränkt sich nicht nur auf die Berücksichtigung von Kunden und ihren Bedürfnissen. Sie kann auf alle Anspruchs- bzw. Stakeholder-Gruppen, die mit dem Unternehmen in Kontakt stehen, ausgeweitet werden. Hinzu kommen - eine spezielle Perspektive der Nachhaltigkeitsdiskussion - auch nicht unmittelbar präsente Anspruchsgruppen wie die nachfolgenden Generationen oder die Bevölkerung der Dritten Welt.

Wie aufwändig deshalb „Nachhaltigkeitsanalysen“ sein können, läßt sich am Corporate Responsibility-Rating von ökom research veranschaulichen. Die Bewertung der Verantwortung eines Unternehmens orientiert sich an den drei Dimensionen des Frankfurt-Hohenheimer Leitfadens: Kulturverträglichkeit, Sozialverträglichkeit und Naturverträglichkeit. Hierzu erfolgt die Auswertung von Unternehmensinformationen und Sekundärliteratur, ein Fragebogen, Interviews von Mitarbeitern, Recherche bei unabhängigen Experten (2).

Der Versuch solcher Ratings und der damit verbundene Aufwand ist sicherlich hoch einzuschätzen, insbesondere hinsichtlich daraus möglicher Anstöße für eine nachhaltige Entwicklung. Auf jeden Fall helfen sie, die Entwicklung der sozialen Dimensionen analog der Fortschritte bei den ökologischen voranzutreiben, indem auf Unternehmensebene dazu angeregt wird, systematisch die verschiedenen Stichworte in den Fragebögen und den zu überprïfenden Themenbereichen durchzugehen. 


\section{- Kommunikation als Lernprozess}

Als Problematik ist damit allerdings verbunden, dass viele Unternehmen sich bemühen, gezielt ihr Image aufzubessern. Entsprechend besteht die Versuchung, das Ganze an Bevollmächtigte - Umweltbeauftragte, Frauenbeauftragte, Nachhaltigkeitsbeauftragte - zu delegieren, die dafür verantwortlich zeichnen. Ihre Aufgabe kann sich schnell darauf beschränken, die verschiedenen Stichworte systematisch zu bearbeiten und ,abzuhaken“. Auf diese Weise kristallisiert sich eine gewisse mechanistische Arbeitsweise heraus, die sich in der Auseinandersetzung mit Checklisten, Fragebögen, Ratingverfahren oder Audits mehr oder weniger zwangsläufig einstellt. Das heißt, es wird vorrangig an einzelnen betrieblichen Symptomen gearbeitet, ohne das externe Umfeld oder auch das einzelne Unternehmen tatsächlich im Sinne einer lernenden Organisation nachhaltiger zu gestalten.

Welche Vorgehensweise könnte hier vielleicht bessere Ergebnisse bringen? Ein wichtiger Aspekt sozialer Nachhaltigkeit ist die Verminderung von Konfliktpotenzialen bzw. deren Nutzung für soziale Innovationen, um so soziale Energien freizusetzen, die in gemeinsame Entwicklungen münden. Hierfür wäre eine stärker prozess- und kommunikationsorientierte Vorgehensweise Erfolg versprechender. Wie aber lassen sich für die Vielzahl von Konfliktfeldern Akteure finden, die von den Maßnahmen des Unternehmens besonders betroffen sind und/oder ein besonderes Interesse an zukunftsorientierten Formen nachhaltiger Entwicklung zeigen?

\section{- Wege zum konstruktiven Dialog}

Entsprechender Sachverstand und Interesse existieren nicht zuletzt bei der Vielzahl der Nichtregierungsorganisationen (NGOs). Fast zu jedem Bereich gibt es NGOs, die für Produkte der entsprechenden wirtschaftlichen Branche soziale Problemfelder und Möglichkeiten zu deren Bearbeitung kompetent benennen können. Über ihre gezielte Einbindung ließe sich verstärkt ein öffentlicher Dialog zu einzelnen Produkten oder Produktionsverfahren fördern.

Ziel solcher Dialoge wäre ähnlich wie bei ökologischen Problemen nicht vorrangig das Aufdekken schon vorhandener Mängel und deren Beseitigung oder Kompensation im Sinne sozialer Nachsorge. Vielmehr gilt es Foren der sozialen Vorsorge zu kreieren und sie einzurichten, bevor ein Produkt auf dem Markt kommt.
Dies erfordert, ein differenziertes Kaleidoskop geeigneter Kommunikationsformen zu nutzen, aber auch weiter zu entwickeln. Erfahrungen und Instrumente aus dem Spektrum politischer Beteiligungsverfahren wie Bürgeranhörung, Planungszelle oder Zukunftswerkstatt müssten hier angepasst zum Einsatz kommen. Als Ergänzung oder Verstetigung solcher eher fallbezogenen Dialoge könnte auch deren Institutionalisierung versucht werden, indem Unternehmen entsprechend Beiräte einrichten oder Vertreter relevanter gesellschaftlicher Gruppen in ihren Aufsichtsrat berufen.

Ergebnis der gezielt organisierten Kommunikation mit den Stakeholdern könnte ein ökologisches und soziales Produktdesign sein, das die entsprechend ausgerichtete Multifunktionalität von Produkten und Produktionsweisen in den Mittelpunkt rückt. Für Widersprüche zwischen ökologischen und sozialen Anforderungen wären dann gemeinsam Lösungen zu entwickeln. Ein Problem bei solchen dialogintensiven Gesprächskreisen und Partizipationsformen bleibt der Widerspruch zur dritten Dimension nachhaltigen Wirtschaftens, der Wirtschaftlichkeit. Trotz aller Chancen, die für die beiden anderen Dimensionen in einer solchen Vorgehensweise stecken, muss eine solche Einbindung der Stakeholder für das einzelne Unternehmen in einem ökonomisch sinnvollen Aufwands- und Ertragsverhältnis stehen.

\section{Anmerkung}

(1) Deutlich wurde dies auch auf dem zweiundzwanzigsten Sommerseminar des Theoriearbeitskreises Alternative Ökonomie (TAK AÖ). Der Beitrag gibt einige Diskussionsstränge einer viertägigen Arbeitsgruppe zu diesem Thema wieder.

(2) Vgl. zum Corporate Responsibility Rating auch

Reinhard, Dirk: Autobranche im Nachhaltigkeitsstau? In Ökologisches Wirtschaften 6/00, S. 6.

\section{Die Autoren}

Dr. Burghard Flieger ist als Dozent, Forscher, Redakteur und Berater im Genossenschaftswesen tätig. Kontakt: Erwinstraße 29, 79102 Freiburg, Tel. 0761/ 709023, Fax 0761/ 709084.

E-mail: genossenschaft@t-online.de

Elmar Sing arbeitet als Unternehmensberater mit den Schwerpunkten Konfliktmanagement, Team- und Personalentwicklung. Er ist Vorstandsmitglied des Unternehmensverbands UnternehmensGrün.

Kontakt: Frankenberger Weg 9, 68309 MannheimVogelstang, Tel. 0621/ 7992487.

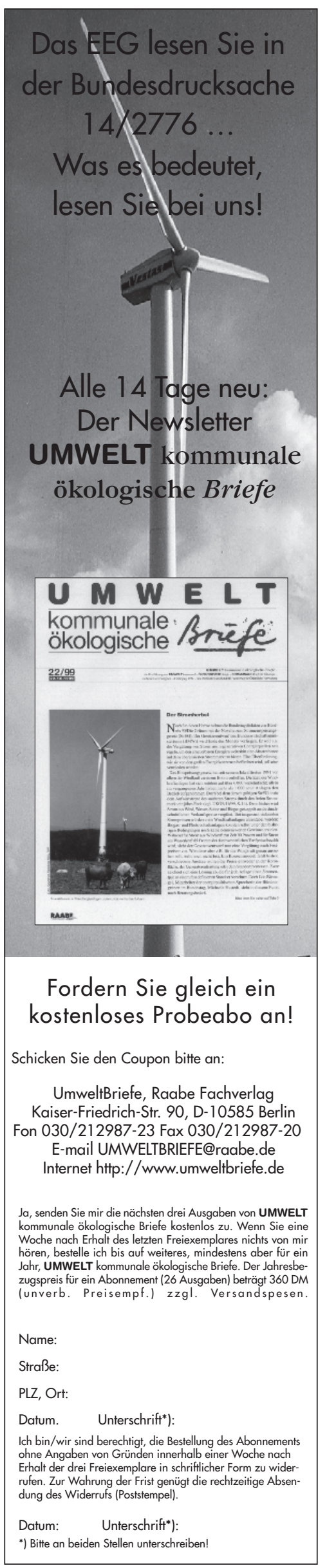


(c) 20I0 Authors; licensee IÖW and oekom verlag. This is an article distributed under the terms of the Creative Commons Attribution Non-Commercial No Derivates License (http://creativecommons.org/licenses/by-nc-nd/3.o/), which permits unrestricted use, distribution, and reproduction in any medium, provided the original work is properly cited. 